\title{
ADAPTIVE HANDOVER HYSTERESIS AND CALL ADMISSION CONTROL FOR MOBILE RELAY NODES
}

\author{
Battulga Davaasambuu $^{1}$, Frank Semaganga ${ }^{2}$ and Takuro Sato ${ }^{2}$ \\ ${ }^{1}$ Graduated School of Global Information and Telecommunication Studies, Waseda \\ University, Bldg. No. 29-7, 1-3-10 Nishiwaseda, Tokyo, Japan 169-0051 \\ ${ }^{2}$ Department of Communications and Computer Engineering, School of Fundamental \\ Science and Engineering, Waseda University, 66 Bldg., 802, 3-14-9 Ookubo, Tokyo 169- \\ 0072, Japan
}

\begin{abstract}
The aim of equipping a wireless network with a mobile relay node is to support broadband wireless communications for vehicular users and their devices. The high mobility of vehicular users, possibly at a very high velocity in the area in which two cells overlap, could cause the network to suffer from a reduced handover success rate and, hence, increased radio link failure. The combined impact of these problems is service interruptions to vehicular users. Thus, the handover schemes are crucial in solving these problems. In this work, we first present the adaptive handover hysteresis scheme for the wireless network with mobile relay nodes in the high-speed train scenario. Specifically, our proposed adaptive hysteresis scheme is based on the velocity of the train. Second, the handover call dropping probability is reduced by introducing a modified call admission control scheme to support radio resource reservation for handover calls that prioritizes handover calls of mobile relay over the other calls. The proposed solution in which adaptive parameter is combined with call admission control is evaluated by system level simulation. Our simulation results illustrate an increased handover success rate and reduced radio link failures.
\end{abstract}

\section{KEYWORDS}

Handover, hysteresis, mobile relay node; call admission control, LTE

\section{INTRODUCTION}

Long-Term Evolution (LTE) is the wireless technology that was developed by the 3rd Generation Partnership Project (3GPP) and which contributed to the development of $4 \mathrm{G}$ systems. LTE supports the OFDM (orthogonal frequency-division multiple), 2x2 and 4x4 MIMO (multi-input multi-output) antenna systems, as well as FDD (frequency-division duplexing) and TDD (timedivision duplexing) modes. Apart from providing cost-effective broadband communication services, LTE provides improved capacity, higher throughput, increased spectrum efficiency, lower latency and improved coverage [1]. LTE has been superseded by enhanced LTE, which is known as Long-Term Evolution Advanced (LTE-A). LTE-A includes new capabilities, which offer sufficient performance to support good-quality IP-based video streaming and other multimedia services over IP to a large number of customers simultaneously [1]. 
A relay node architecture is included in LTE with the purpose of increasing the capacity of the system and the coverage area [2,3]. The relay node architecture is schemed to support mobility in LTE-A Release 12 [4,5]. From a technical point of view, similar to the fixed relay node (FRN), the mobile relay node (MRN) is connected to the Donor Evolved Node B (DeNB) node via a radio interface backhaul link (named as Un) for data transmission. Moreover, to exchange control information, the MRN is associated to a mobility management entity (MME) and Evolved Universal Terrestrial Radio Access (E-UTRAN). In addition, the user equipment (UE) is connected to an MRN located either in the vehicle or in a public transportation vehicle, such as a train or bus. Figure 1 contains a diagram showing the MRN architecture. The passengers in the vehicle do not use handover (HO) between the base stations; MRN only executes HO by using the outer antenna of the vehicle [4]. The two main problems associated with MRN HO procedures are that: (1) they require radio resources to be reserved for the MRN HO call; and (2) they need to be instructed on how to finish $\mathrm{HO}$ procedures of the train before losing the signal of the serving cell.

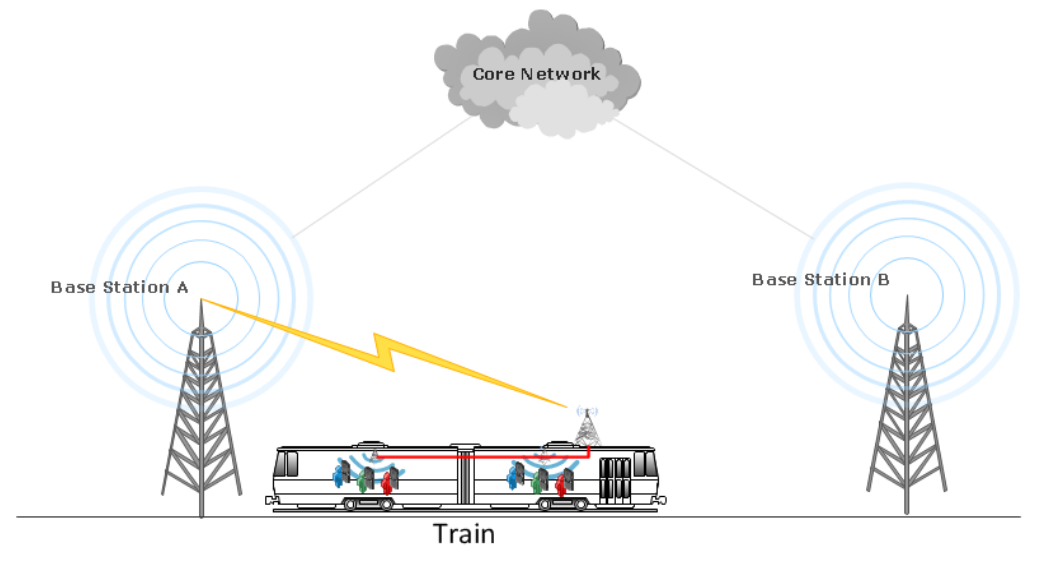

Figure 1. A system model of the mobile relay node (MRN).

In LTE systems, call admission control (CAC) functions to manage radio resources, as well as call blocking or acceptance. When admitting calls, CAC can either process a new call or an HO call based on the availability of resources in the target cell. The radio resources are efficiently utilized, and the quality of service (QoS) in terms of UE requirements is satisfied. In addition, the target cell should provide resources for the MRN and its connected UE during the HO procedure and cell residence time. Therefore, our proposed CAC scheme is required to realize radio resource reservations for three call types (a new call, UE HO call and MRN HO call) and to minimize the dropping of MRN HO connections simultaneously. If CAC blocked the MRN HO call, MRN would have to re-initiate the HO procedure to the other DeNBs. This, in turn, requires more time for $\mathrm{HO}$, and as a result, a further delay and increased $\mathrm{HO}$ failure rate is introduced.

The main part of mobility management for MRN is the HO scheme that can be used to support seamless connectivity for a mobile terminal moving into the overlap area. The HO of the LTE system with MRN is based on network-controlled hard handover ( $\mathrm{NCHH}$ ) [4]. The hard $\mathrm{HO}$ is a break-to-make method, in which old link between the MRN and the source eNodeB is released before the new link (between the MRN and the target eNodeB) is established. Otherwise, MRN $\mathrm{HO}$ reuses conventional $\mathrm{NCHH}$. It is well known that the typical $\mathrm{HO}$ process consists of preparation, execution and completion [6]. Conventional NCHH is more satisfactory in a lowmobility environment. Why is it not satisfactory for use with a high-speed mobility environment? High-speed mobility in the overlap area has the advantage of a reduced ping-pong $\mathrm{HO}$ ratio for the passengers of the vehicles, but it also imposes disadvantages resulting from an increased radio link failures (RLF) ratio, service interruption and a reduced HO success rate. In contrast, low- 
speed mobility causes UEs to incur a high ping-pong $\mathrm{HO}$ ratio and a low RLFs ratio. An enhanced $\mathrm{HO}$ scheme is therefore crucial to solving these problems. Our proposed HO scheme would enable a reduction in RLF and would increase the probability of HO success in a highspeed mobility environment.

The main aim of this paper is to propose an enhanced HO scheme capable of dynamically tuning $\mathrm{HO}$ hysteresis $(\mathrm{HH})$ based on the velocity of the train. $\mathrm{HH}$ is the main parameter that can manage the starting point of the $\mathrm{HO}$ procedure in the preparation step. Then, to reduce the HO dropping probability (HDP), we reverse the radio resources for the MRN HO procedure.

Wireless networks based on a CAC scheme have been described and studied in many papers. In [11], the authors analyzed the adaptive resource allocation scheme for two cases, namely with both non-priority and guard-channel schemes for the adaptive HO scheme, and the corresponding simulation results showed that adaptive schemes are able to reduce the dropping probability of HO connections. In [12], the authors introduced an adaptive CAC algorithm to complement the resource reservation mechanism with a bandwidth reallocation policy. The proposed scheme was shown to significantly reduce the probability of new call blocking and HO call dropping. The self-optimization of the CAC is being investigated in the SOCRATES (Self-Optimisation and self-ConfiguRATion in wirelEss networkS) project [13] for LTE and next generation mobile networks.

The HO for MRN is introduced similarly to a regular UE HO (which is named NCHH) in 3rd Generation Partnership Project (3GPP) Technical Report 36.836 [4]. Researchers introduced an HO scheme for relay-based networks. Chen proposed an HO-based relay enhanced for UE [7]. They proposed a novel HO scheme based on a cost function, including signal cost and throughput on LTE cellular relay networks, and compared their scheme with conventional NCHH of an LTE network. Moreover, [8] introduced a cell selection mechanism for mobile relay operations based on the geometric boundary of the cell and the obtained location of the UE. The analysis and simulation results presented in [3] are related to moving relays and mobility optimization for relay-enhanced networks. In addition, RSS-based HO was introduced between the serving DeNB and the target DeNB for LTE relay architecture alternatives, Alt1 and Alt2 [5]. A cost-based vertical HO algorithm decision with a combination of the prediction of SINR (signal to interference plus noise ratio) in heterogeneous wireless networks has been demonstrated [9]. A cost function was introduced on the basis of multi-attribute QoS, and an approach for optimal network selection was introduced. In [10], Thomas researched the relationship between hysteresis and time-to-trigger and presented an adaptive $\mathrm{HO}$ scheme based on $\mathrm{HO}$ performance indicators.

First, we investigate an adaptive $\mathrm{HH}$ scheme based on the train's velocity to increase the HO performance of hard HO in an LTE system. The aim of the adaptive HH scheme, which is based on the speed of the vehicle with MRN, is to influence the HO triggering. Moreover, the proposed $\mathrm{HO}$ can increase the time for the $\mathrm{HO}$ procedure, including the time required by the security policy and data re-routing, when the velocity of the vehicle is high in the overlap area. Second, we propose a CAC scheme to reduce the dropping ratio of MRN HO calls. The aim of the proposed $\mathrm{CAC}$ is to support radio resource reservation for MRN HO calls.

\section{ADAPTIVE HANDOVER HYSTERESIS}

The MRN is always controlling Reference Signal Received Power (RSRP) of the serving DeNB. When MRN reaches the boundary of the serving DeNB or the designated criteria, the measurement is performed and sent to the serving DeNB. The candidate target DeNBs in this measurement report are related to the serving DeNB by utilizing the X2 interface to estimate the load of all of the target cells and then to generate a priority list based on load. The cell boundary is the starting point of overlap between the neighbour cells. 
Figure 2 shows the calculation of the HH value when an MRN moves to the target DeNB. In this calculation of the $\mathrm{HH}$ value, $H_{\text {def }}$ is the default $\mathrm{HH}$ value configured by the system, $\Delta H$ denotes the adjustment value of $\mathrm{HH}$ calculated by a function and $H_{\max }$ is the maximum $\mathrm{HH}$ value. If the calculated hysteresis exceeds $H_{\max }$, the serving DeNB sends a command to the MRN requesting a new hysteresis calculation and the target DeNB. The point at which the RSRP value of the target DeNB exceeds the value of the serving DeNB is named minimum hysteresis. A selected $\mathrm{HH}$ can provide efficient $\mathrm{HO}$ decisions and triggering that can manage a starting point of the $\mathrm{HO}$ procedure in the preparation step of the hard handover procedures. In the proposed scheme, the $\mathrm{HH}(H)$ value is automatically adjusted and calculated by Equation 1.

$$
H=H_{\text {def }}-\Delta H
$$

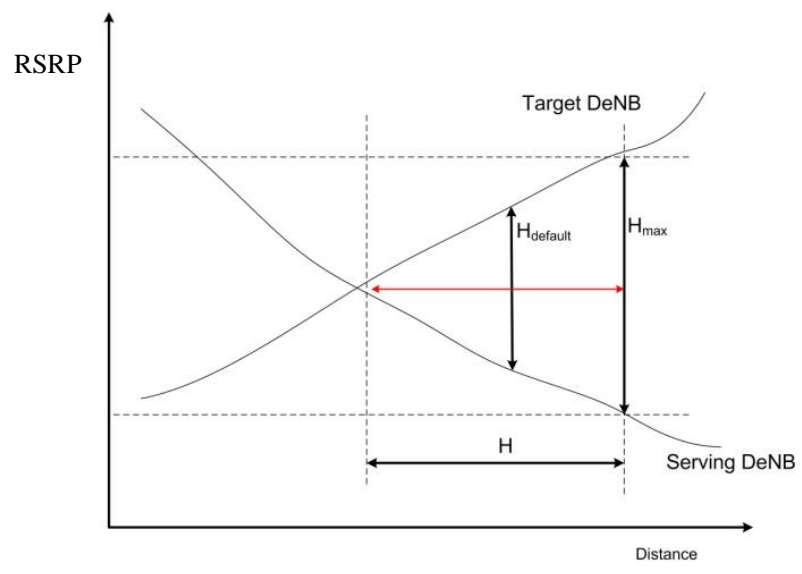

Figure 2. Hysteresis values when an MRN moves.

Moreover, if the situation represented below (Equation 2) occurs, the MRN starts an HO procedure for the target DeNB.

$$
R_{\text {serving }}-R_{\text {target }} \geq H
$$

where $R_{\text {target }}$ and $R_{\text {serving }}$ denote the RSRPs of the target DeNB and serving DeNB, respectively. In (7), $\Delta H$ is expressed by:

$$
\Delta H=\alpha * f_{H}
$$

where $\alpha$ selects less than $H_{\max }-H_{\text {def }}$. If $\alpha$ is increased, the range of $\Delta H$ will be extended, in which case it would be more agreeable to find the best $\mathrm{HH}$ value for the $\mathrm{HO}$ procedure. A $f_{H}$ is the function that are presented in Equation 4.

$$
f_{H}=\log _{H_{\text {def }}} \frac{\left(V_{\max }-V_{\text {current }}+1\right)}{V_{\max }}
$$

where $V_{\text {current }}$ is the current velocity of the MRN, $H_{\text {def }}$ is the default fixed value of the hysteresis and $V_{\max }$ is the maximum velocity of the MRN $(=350 \mathrm{~km} / \mathrm{p})$. If $V_{\text {current }}$ were to reach maximum velocity, $N_{\text {velocity }}$ would be equal to $H_{\text {def }}$. Thus, the HO decision (or time-to-trigger) would shift depending on the velocity of the MRN. 


\section{CALL ADMISSION CONTROL}

The serving DeNB send the "HO request" with context information to the target DeNB, if Equation 2 keep during the preparation step of the HO procedure. The "HO request" with context information include of the number of MRNs outside antennas and the connected devices of passengers. After handling the "HO request" on the target DeNB, the CAC scheme allocates a radio resource for the received request. The resource management consists of $\mathrm{CAC}$, the efficient utilization of power and a channel allocation strategy. CAC denotes the process of admitting a new call or an $\mathrm{HO}$ call, based on the availability of resources in the target cell. The utilization of power controls the transmission power between the target cell and the MRN. Moreover, the target cell classifies calls when a connection request arrives. In CAC, two important QoS parameters are the new call blocking probability (NCBP) and handover call dropping probability (HCDP), both of which apply during the HO procedure. The NCBP is the probability of dropping a new call during the $\mathrm{HO}$ procedure, whereas HCDP is the probability of $\mathrm{HO}$ failure or dropped $\mathrm{HO}$ calls by the target cell. If the available resources are insufficient in the target cell for accepting the HO calls, the target cell will drop them. The result of this is an increase in the unsuccessful HO ratio. The typical CAC schemes can reserves the radio resource for $\mathrm{HO}$ calls and the new calls (two classifications) in the target cell. In this point, the HO call of MRN is the same as HO calls of UEs.

In proposed CAC scheme, calls are classified according to three types: $\mathrm{HO}$ calls of MRNs, HO calls of UEs and new calls. Additionally, if HO calls of MRNs in received, CAC need reserve the radio resources for the connected devices of passengers. Moreover, we propose a CAC scheme for the wireless network, which provides a radio resource allocation policy with three classes and prioritizes MRN HO calls over UE HO calls and new calls. The focus of the proposed CAC scheme is to improve the utilization of radio resources in order to reduce the dropping probability of MRN HO calls.

The proposed scheme is based on the guard channels scheme that can reserves channels separated the $\mathrm{HO}$ calls of MRN and $\mathrm{HO}$ calls of UEs. On the other words, the total channels of the target cell are divided into three parts, $\mathrm{HO}$ calls of UEs, HO calls of MRN and new calls. The system model for our scheme is shown in Figure 3. For example, C1-C2 are reserved for $\mathrm{HO}$ calls of UEs, $\mathrm{C} 2-\mathrm{C} 3$ are reserved for $\mathrm{HO}$ calls of MRN and devices of passengers, and the remaining channels (Channel 1-C1) are shared by all calls.

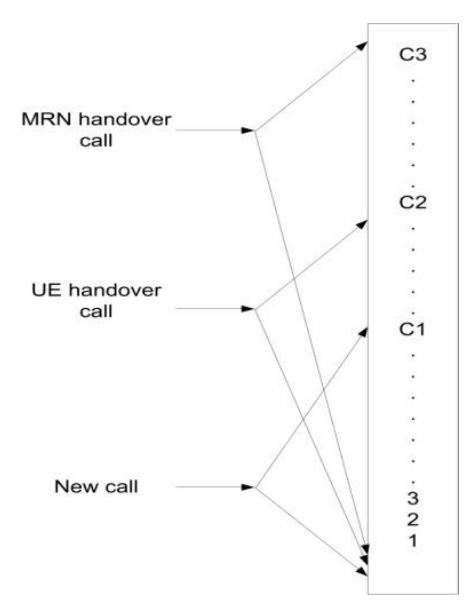

Figure 3. A system model of $\mathrm{C}$ channels. 
In the system, the arrival of calls is represented by a Poisson function with the arrival rate of new calls denoted by $\lambda_{n}$, HO calls of UEs by $\lambda_{\text {ue }}$, and HO calls of MRNs with devices of passengers by $\lambda_{m r m} \cdot \lambda_{m r m}$ denotes a channel number of MRN and the connected devices of passengers. In other words, if $\mathrm{HO}$ calls of MRN is received, the reserved radio resources and the number of the connected devices is equal in the proposed CAC. The call holding time for all three types of call is exponentially distributed with $1 / \mu, 1 / \eta$ and $1 / \beta$, respectively. Let $\rho=\left(\lambda_{n}+\mu\right)\left(\lambda_{u e}+\eta\right)($ $\left.\lambda_{m m}+\beta\right)$ be the total arrival rates of calls to the target cell. If the target cell has $n$ channels, let $P_{n}$ be the probability. The probability $P_{n}$ that $n$ channels are busy is:

$$
P_{n}=\left\{\begin{array}{cc}
\left(\frac{\rho^{n}}{n !}\right) P_{0} & 0 \leq n \leq C 1 \\
\rho^{C 2}\left(\frac{\lambda_{u e}{ }^{n-C 2}}{n !}\right) P_{0} & C 1 \leq n \leq C 2 \\
\rho^{C 3}\left(\frac{\lambda_{m m n}{ }^{C 2-C 3}}{n !}\right) P_{0} & C 2 \leq n \leq C 3
\end{array}\right.
$$

where:

$$
P_{0}=\left\lfloor\sum_{n=0}^{c 1} \frac{\rho^{n}}{n !}+\rho^{C 2} \sum_{n=C 1+1}^{C 2} \frac{\lambda_{u e}{ }^{n-C 2}}{n !}+\rho^{C 3} \sum_{n=C 2+1}^{c 3} \frac{\lambda_{m r n}{ }^{C 2-C 3}}{n !}\right\rfloor^{-1}
$$

Following which, the MRN HCDP is calculated by using the following:

$$
P_{l_{m r m}}=\sum_{C 2}^{C 3} P_{n}+\sum_{C 1}^{C 2} P_{n}+\sum_{1}^{c 1} P_{n}
$$

When a new call arrives, the target cell will check the channels up to $\mathrm{C} 1$. If channels are not available, the target cell drops the new call it has received. Upon arrival of an $\mathrm{HO}$ call of UE, the target cell first checks the channels between $\mathrm{C} 1$ and $\mathrm{C} 2$. If channels are not available, the target cell checks between 1 and $\mathrm{C} 1$. If it is unable to find an available channel, the $\mathrm{HO}$ call of UE is dropped by the target cell. When an HO call of MRN arrives, CAC gets the number of the connected devices from "HO request" with context information. Then, the target cell checks between $\mathrm{C} 2$ and $\mathrm{C} 3$, and if no channels are available, it again checks between $\mathrm{C} 1$ and $\mathrm{C} 2$. If no channels are available, the target cell checks between 1 and $\mathrm{C} 1$. The result of this procedure is that, because none of the channels of the target cell are free, the HO call of MRN is dropped. Figure 4 describes the procedure of our scheme. 


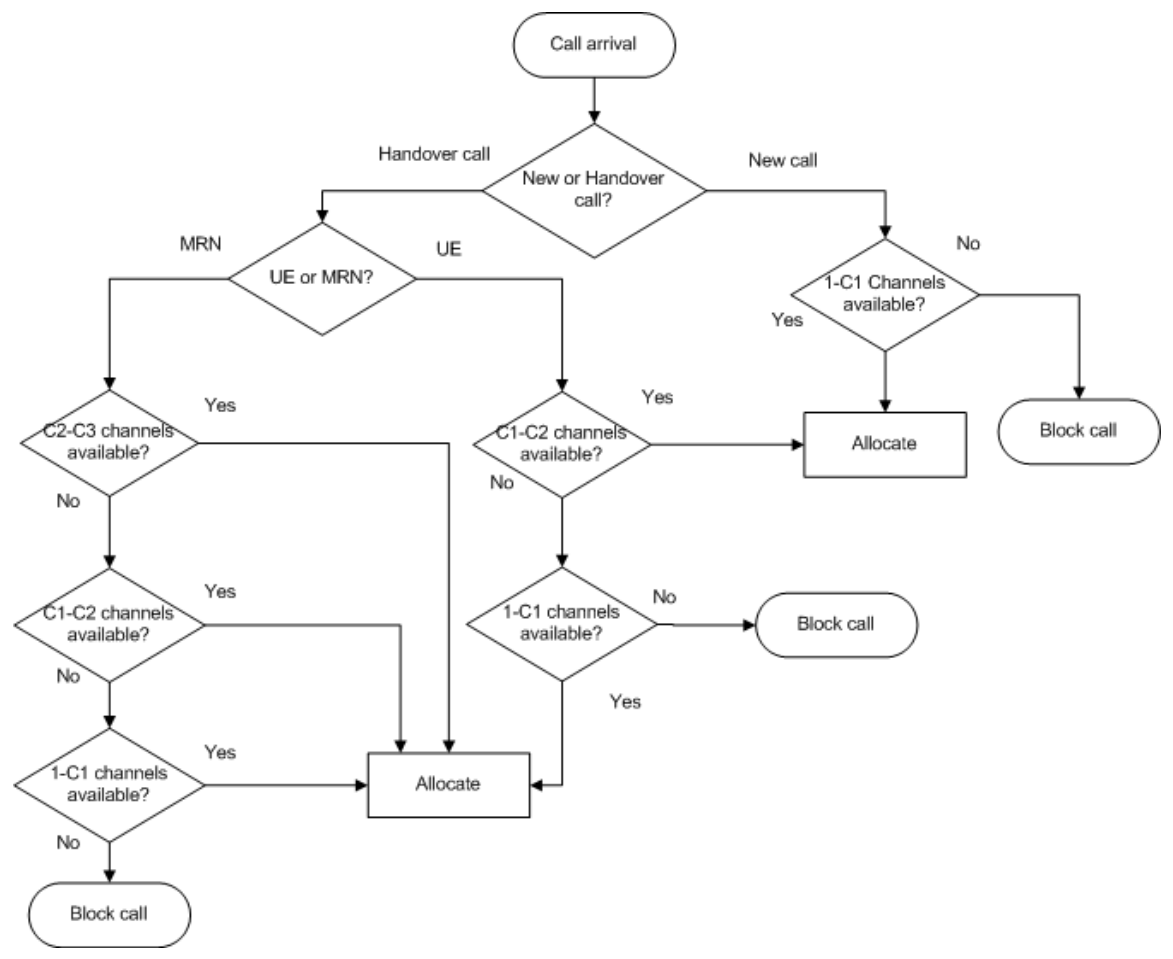

Figure 4. Call admission control (CAC) procedure of our scheme.

\section{SIMULATION RESULTS}

This section presents the simulation of our schemes. First, we simulated an adaptive HO scheme based on the velocity of the vehicle equipped with an MRN. Second, we simulated two CAC schemes, the conventional and the proposed CAC schemes.

\subsection{ANALYSIS OF THE PROPOSED HANDOVER}

Our proposed the adaptive HH scheme is simulated on the open source LTE-sim [15]. For simulation purposes, a connection manager application was used to modify and implement in order to control connection quality between the serving DeNB and the MRN. In addition, we have to modify and add the DeNBs for calculating the load of the target DeNBs by considering sending a request to the target DeNBs to receive the necessary information and to repeat the sorting of the target DeNBs according to their load. Table 1 lists all of the parameters of the simulation.

Figure 5 shows the scenario we used for our simulation. It consists of a homogeneous network with urban cells (the radius is $1 \mathrm{~km}$ ) that includes five LTE eNBs, the MRN-installed vehicle (a train) and its number of connected UEs between 10 and 50. The MRN is equipped with a transparent router between the outside and inside antennas. The cell boundary and overlap of neighbouring cells is 300 meters. At the beginning of the simulation, the MRN is connected to the serving DeNB. At the starting point of the overlap area, the MRN make a measurement report for $\mathrm{HO}$ and send to the serving DeNB. After received this report, the serving DeNB calculates the $\mathrm{HH}$ and to run a cell selection scheme based on the load of the candidate DeNBs. However, the MRN controls the RSRP of the serving and target DeNBs, if the conditions in Equation 2 are fulfilled, by starting time-to-trigger. A simulation can effectively show the difference between the static hysteresis-based conventional HO and the proposed HO with dynamically changed hysteresis based on the speed of the train. 
Table 1. Simulation parameters.

\begin{tabular}{|l|l|}
\hline Parameter & Value \\
\hline Number of Donor eNBs & 5 cell sites, urban area \\
\hline Radius of cell & 1 kilometer \\
\hline Overlap area between two cells & $300 \mathrm{~m}$ \\
\hline $\begin{array}{l}\text { Distance between eNodeB and } \\
\text { railway line (MRN) }\end{array}$ & $100 \mathrm{~m}$ \\
\hline Cell Tx power & $46 \mathrm{dBm}$ \\
\hline Carrier frequency & $2.6 \mathrm{GHz}$ \\
\hline Path loss model & $128.1+37.6 \log _{10} \mathrm{~d}$ \\
\hline Shadow fading deviation & $4 \mathrm{~dB}$ \\
\hline System bandwidth & $10 \mathrm{MHz}$ \\
\hline Transmission time interval & $1 \mathrm{~ms}$ \\
\hline Traffic type & Video stream, VoIP and data \\
\hline MRN speed & Interval $[120 \mathrm{~km} / \mathrm{h}, 350 \mathrm{~km} / \mathrm{h}]$ \\
\hline
\end{tabular}

Figure 6 shows the simulation results of the HO triggering probability with the location of the train equipped with MRN and five different speed scenarios up to $350 \mathrm{~km} / \mathrm{p}$. The $\mathrm{x}$-axis is the distance between the train with the MRN and the serving DeNB. Starting from the location $\mathrm{x}=$ 840, the RSRP of the target DeNB starts to overbear the RSRP of the serving DeNB. The resulting HO triggering probability increases at the location $\mathrm{x}=850$, and the velocity is typically higher rather than lower. Further, the triggering probability increases abruptly up to 90 percent of the distance between the locations $\mathrm{x}=875$ and $\mathrm{x}=925$. Thus, our proposed HO scheme is capable of triggering the probability based on velocity. At the location $\mathrm{x}=850$, the signal quality of the target DeNB is sufficient to meet the communication requirement. In addition, the proposed $\mathrm{HO}$ scheme can provide the $\mathrm{HO}$ decision, and the starting time satisfies the high-speed mobility environment.

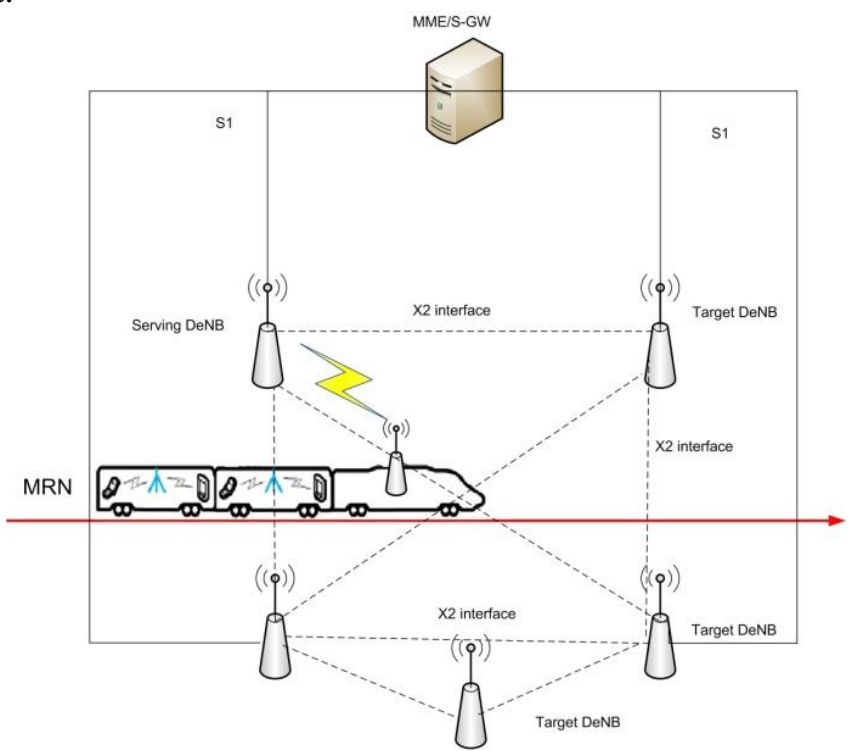

Figure 5. Homogeneous network.

Figure 7 shows the simulation results of the RLF ratio with different MRN velocities in the interval $[120,350]$. In the figure, our proposed $\mathrm{HO}$ scheme with adaptive $\mathrm{HH}$ compares hard HO with the received signal strength based scheme that employs regular UE HO based on static HO parameters, as defined by the 3GPP standard, which has been reused for MRN [4]. In a low mobility environment, the two schemes behave similarly. However, when the MRN runs at high speed, the change in the RLF of the proposed scheme is low. It is also observed that as the speed increases, the proposed scheme to decrease the $\mathrm{HH}$ value. This is because in the proposed scheme 
the train with high-speed required smaller $\mathrm{HH}$ value than the user devices with low-speed. As a result, the $\mathrm{HO}$ procedures start too early. If the train leaves from the coverage of the serving DeNB before the HO procedure was completed, the number of RLFs increase. RLFs is dropped $\mathrm{HO}$ calls before the $\mathrm{HO}$ procedure was completed, if the train leaves the coverage of the serving DeNB. Moreover, the device makes a new connection with the available cell. In other hands, HO procedure failed (not RLFs), MRN must re-connect to the serving DeNB and restart the HO procedure.

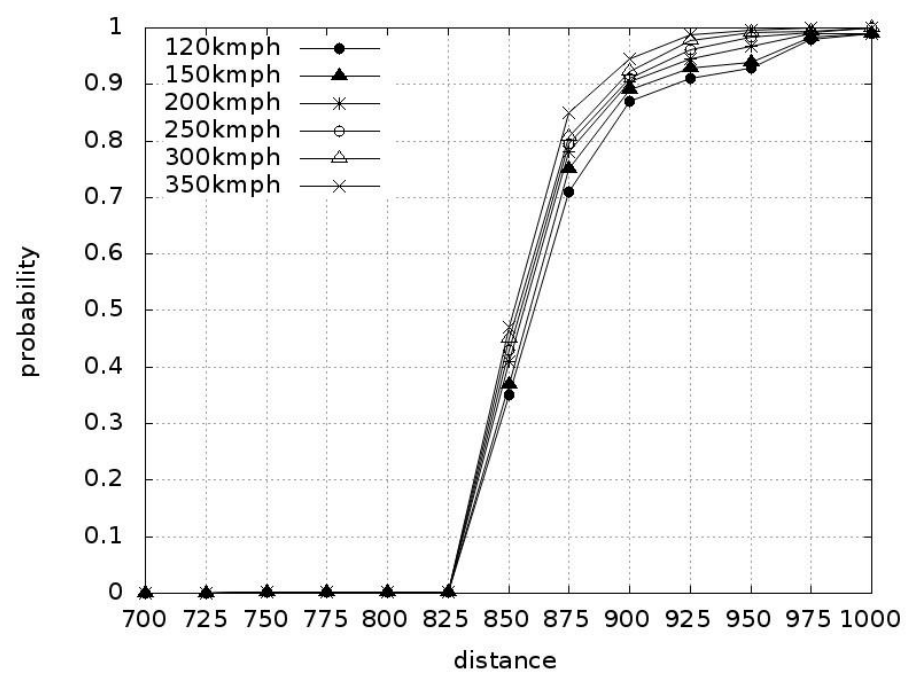

Figure 6. Handover triggering probability of different velocities.

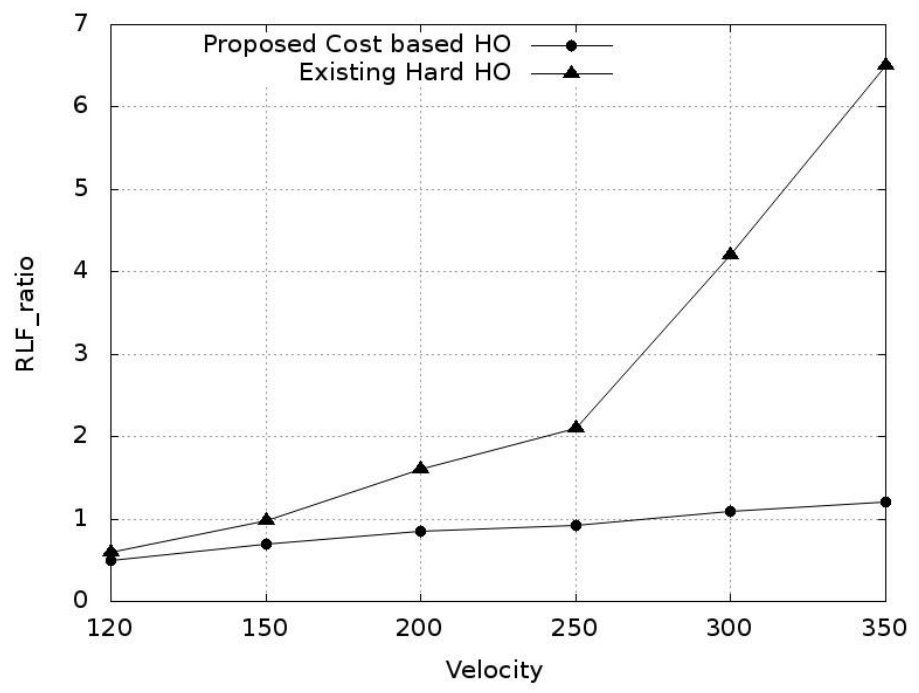

Figure 7. The radio link failure (RLF) ratio of the proposed scheme and the hard handover scheme at MRN velocity.

\subsection{ANALYSIS OF THE CAC SCHEME}

In this subsection, we present our analysis of the effectiveness of the proposed CAC, which performs the task of reducing the $\mathrm{HO}$ dropping probability. We consider three priority classes in the homogeneous system: new calls, HO calls of UEs, and HO calls of MRN. The call arrivals are generated based on Poisson process with a mean arrival rate of (calls/s). Simulation model and parameters are considering Table 1 . We choose a set other parameters: the channel $\mathrm{C} 3$ is the number of all radio resources on the target cell, guard channel C2 is 75 percent of all radio resources, and $\mathrm{C} 1$ is 50 percent of the remaining radio resources. 
Furthermore, the performance is evaluated with/without the effect of the proposed CAC using the following performance metrics: (i) the new call blocking probability is defined as the faction of new calls that are blocked on the target cells; (ii) the UE HO dropping probability is defined as the faction of $\mathrm{HO}$ call of UE attempts that are blocked because of lack of available channels; and (iii) the MRN HO dropping probability is defined as the faction of $\mathrm{HO}$ call of MRN attempts that are blocked because of all channels being unavailable.

Figure 8 shows the difference between the proposed and the conventional CAC schemes. The non-priority based conventional CAC cannot reserve channels for $\mathrm{HO}$ calls. In other words, conventional CAC that used FIFO (first input first out) algorithm and will drop calls (both new calls and HO calls) if all channels are busy [14]. The proposed CAC separated HO calls of MRNs and HO calls of UEs. The "NCBP non-prioritized" and "HCDP non-prioritized" are the NCBP and HCDP when conventional CAC was active, respectively. The "NCBP proposed", "HCDP proposed" are NCBP and HCDP of MRN and its connected UEs when the proposed CAC was active, respectively. The $\mathrm{y}$-axis represents the call blocking probability between zero and 25 percent.

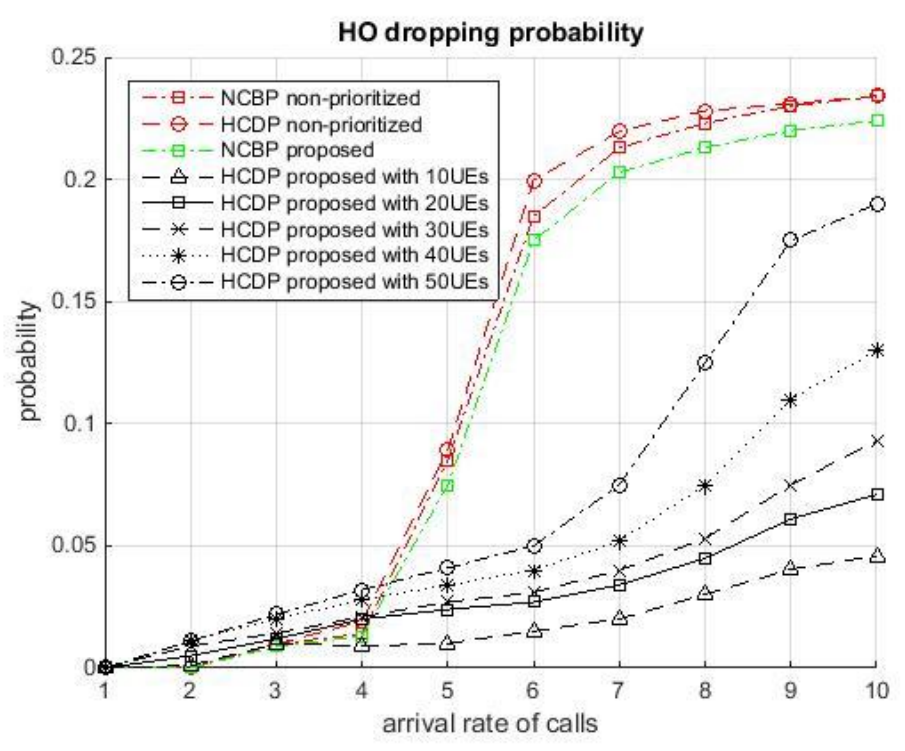

Figure 8. HCDP. The connected UEs of MRN is an interval between 10 and 50.

From figure, we observe the new call blocking probability and the handover call dropping probability increase as the arrival rate increases. The blocking and dropping probability of the conventional CAC are same during the simulation time. The change in the NCBP of the proposed scheme is low. Furthermore, HCDP of MRN is lower than NCBP because of the guard channels and high priority reservation for HO calls of MRNs. In addition, HCDP of MRN is increased as the number of connected UEs increases because of the CAC scheme need reserve larger radio resources for the larger number of the connected UEs. In the proposed CAC, the new calls are the lowest priority. The results of proposed CAC can be explained by the processes of the proposed scheme: radio resource reservation, three call classification and giving the high priority to HO calls of MRN in the admission step. In addition, our proposed scheme prioritizes HO calls of MRN over HO calls of UEs and new calls (HO calls of MRN are high; HO calls of UEs are medium; and new calls are low). The HO call of MRNs was the same as the HO calls of UEs in the conventional CAC scheme. From this point of view, conventional CAC can reserve one radio resource for MRN. Therefore, the CAC scheme would need to support MRN and its connected devices. To address this need, our proposed CAC reserved the radio resources for MRN and its connected UEs. 


\section{CONCLUSION}

This paper presents an adaptive HH scheme and CAC for MRN in a 3GPP LTE-A system. First, we introduced an adaptive HH scheme between the DeNBs in the LTE network with NCHH. Our proposed scheme focused on adaptive $\mathrm{HH}$ of a hard $\mathrm{HO}$ based on MRN velocity. We compared our proposed scheme with the static HH based NCHH scheme in terms of RLF ratio and HO triggering probability. The result of the simulation showed that our proposed $\mathrm{HO}$ scheme has an increased success rate during $\mathrm{HO}$ procedures. Subsequently, we compared the performance of the proposed CAC scheme and conventional scheme. Our proposed CAC scheme was shown to reduce the $\mathrm{HO}$ call blocking ratio. In addition, the proposed scheme can reserve the radio resources for MRN and its connected UEs.

\section{ACKNOWLEDGEMENTS}

The authors would like to thank everyone, just everyone!

\section{REFERENCES}

[1] Tran, T.T.; Shin, Y.; Shin, O.S. Overview of enabling technologies for 3GPP LTE-advanced. EURASIP Journal on Wireless Communications and Networking 2012, pp. 1-12.

[2] 3GPP. Evolved Universal Terrestrial Radio Access (E-UTRA), Relay architecture for E-UTRA (LTEA). TR 36.806, 3rd Generation Partnership Project (3GPP), 2010.

[3] Christian Pietsch, Stefan Brueck, M.F.T.M.M.K.J.V.M.D.N.E.S.T.S.A.P.Y.S.M. Moving Relays and Mobility aspects. Technical report, ARTIST4G project, 2012.

[4] 3GPP. Study on Mobile Relay for Evolved Universal Terrestrail Radio Access (E-UTRA). TR 36.836, 3rd Generation Partnership Project (3GPP), 2013.

[5] Krendzel, A. LTE-A Mobile Relay Handling: Architecture Aspects. Wireless Conference (EW), Proceedings of the 2013 19th European. VDE, 2013, pp. 1-6.

[6] Kuang, Q.; Belschner, J.; Bleicher, Z.; Droste, H.; Speidel, J. A measurement-based study of handover improvement through range expansion and interference coordination. Wireless Communications and Mobile Computing 2014.

[7] Muqiong, C.; Hong, J.; Xi, L. Handover improvement based on cost function and user fairness for cellular relay enhanced LTE networks. High Technology Letters 2011, vol. 17, pp. 299-304.

[8] Teyeb, O.; Kazmi, M.; Mildh, G. Cell Selection Mechanism in Mobile Relay Operation; Google Patents, 2012. US Patent App. 13/499,952.

[9] Zhang, Y.; Zheng, Z.; Chen, L. A Cost-Based Vertical Handoff with Combination Prediction of SINR in Heterogeneous Wireless Networks. Journal of Theoretical and Applied Information Technology 2013, vol. 49.

[10] Jansen, T.; Balan, I.; Turk, J.; Moerman, I.; Kurner, T. Handover parameter optimization in LTE selforganizing networks. Vehicular Technology Conference Fall (VTC 2010-Fall), 2010 IEEE 72nd. IEEE, 2010, pp. 1-5.

[11] Lee, J.; Kim, H.c. Performance Analysis of Adaptive QoS Handoff Mechanism Using Service Degradation and Compensation. International Journal of Software Engineering \& Its Applications 2013, vol. 7.

[12] Sanabani, M.; Shamala, S.; Othman, M.; Desa, J. Adaptive call admission control for prioritized adaptive services in wireless/mobile multimedia cellular networks. IJCSNS 2006, vol. 6,pp. 115.

[13] Spaey, K.; Sas, B.; Blondia, C. Self-optimising call admission control for LTE downlink. COST 2100 TD(10)10056, Joint Workshop COST 2100 SWG 3.1 \& FP7-ICT-SOCRATES 2010.

[14] Bejaoui, Tarek, and Nidal Nasser. "Handover and class-based Call Admission Control policy for 4Gheterogeneous mobile networks." Computer Systems and Applications. AICCSA. IEEE/ACS International Conference on. IEEE, 2008.

[15] Giuseppe Piro, Luigi Alfredo Grieco, Gennaro Boggia, Francesco Capozzi, and Pietro Camarda", Simulating LTE Cellular Systems: an Open Source Framework", IEEE Trans. Veh. Technol., vol. 60, no. 2, Feb, 2011, doi: 10.1109/TVT.2010.2091660 


\section{AUTHORS}

Battulga Davaasambuu received the B.S. and M.S. degrees from National University of Mongolia, Ulaanbaatar, Mongolia, in 2007 and 2009, respectively. From 2009 to 2011, he worked as a research engineer at the National University of Mongolia. From 2012, he is a Ph.D. candidate at Graduate School of Global Information and Telecommunication Studies (GITS), Waseda University, Tokyo, Japan. He is a student member of IEEE. His research interests include mobile communication, mobility management, next generation wireless networks and their architecture.

He received his BSc. in Telecommunications Engineering from University of Dar es salaam,Tanzania in 2007. He then worked with Telecommunication Companies for 3 years in areas of Network Management and Product Management. In march,2011 He was granted Japanese Government Scholarship. He was a research student at Waseda University from April 2011 to March 2012.He obtained Msc. in Global Information and Telecommunication Studies from Waseda University,Japan in 2014.He is currently doing his $\mathrm{PhD}$ at Waseda University,Japan and his current research interest include RF-Impairments in Massive MIMO Systems, wireless communication in high mobility environment and Millimeter Wave Wireless Communications.

Takuro Sato received the B.E. and Ph.D. degrees in electronics engineering from Niigata University, Niigata, Japan, in 1973 and 1993, respectively. He joined the Research and Development Laboratories, OKI Electric Industry Co., Ltd., Tokyo, Japan, in 1973, where he was a Senior Research Manager and Research Director with Communication Systems Laboratory. He joined the Niigata Institute of Technology from

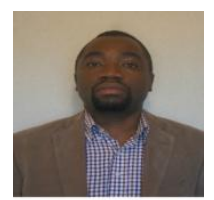
1995 as a Professor where he research focused on CDMA, OFDM, personal communication systems and other related areas. In 2004, he joined GITS, Waseda University, Tokyo, as a professor and currently serving as the Dean. He has engaged in the research on PCM transmission equipment, mobile communications, data transmission technology, and digital signal processing technology. He has developed wideband CDMA system for personal communication system and joined the PCS standardization committee in the USA and Japan. His contributes are mainly in high speed cellular modem standardization for ITU, 2.4GHz PCS for ITA and wireless LAN technology for IEEE 802.11, etc.. He has authored 11 Books and more than 200 papers. His current research interests include Next generation mobile communications, wireless communications, ICN/CCN technology, ICT in Smart Grid and their global standardization. He is a fellow of IEICE and IEEE. 